\title{
Shouting Strengthens Maximal Voluntary Force with Pupillary Dilation
}

\section{Yudai Takarada ( $\nabla$ takarada@waseda.jp )}

Waseda University

Daichi Nozaki

University of Tokyo

\section{Research Article}

Keywords: human maximal voluntary force, neural inhibition, Pupillary Dilation, neuromodulatory

Posted Date: May 27th, 2021

DOI: https://doi.org/10.21203/rs.3.rs-549858/v1

License: (c) (i) This work is licensed under a Creative Commons Attribution 4.0 International License. Read Full License 


\section{Abstract}

Previous research has demonstrated that human maximal voluntary force is generally limited by neural inhibition. Indeed, producing a shout during maximal exertion efforts enhances the force levels of maximum voluntary contractions. However, the mechanisms underlying this enhancing effect of force production remain unknown. We investigated the influence of a shout on the pupil-linked neuromodulatory system state by examining pupil size. We also examined its effect on the motor system state by examining motor evoked potentials in response to transcranial magnetic stimulation applied over the contralateral primary motor cortex, and by evaluating the handgrip maximal voluntary force. Analysis showed that a shout significantly increased the handgrip maximal voluntary force, followed by an increase in pupil size and a reduction of the cortical silent period. Our results indicate that a shout can increase handgrip maximal voluntary force through the enhancement of motor cortical excitability, possibly via the enhancement of noradrenergic system activity. This study provides evidence that the muscular force-enhancing effect of a shout during maximal force exertion is related to both the motor system state and the pupil-linked neuromodulatory system state.

\section{Introduction}

Maximal voluntary contraction (MVC) is the maximal force-generating capacity of a muscle or group of muscles in humans. The MVC is believed to be limited by inhibitory mechanisms. This hypothesis is based on experimental results showing that the MVC is enhanced by manipulations, such as the sound of a gunshot (1), hypnotic suggestion (1), a shout $(1,2)$, and verbal encouragement (3), during maximal exertion efforts. These results indicate that maximum volition-induced motor system activity does not drive muscles to produce the full force of which they are capable and suggest that there is a latent ability for producing additional force that is hidden in ordinary force exertion. If this is true, the aforementioned MVC-enhancing manipulations are likely to enhance the excitability of the motor system. This hypothesis is supported by recent evidence that the subliminal priming of an action concept with a positive reward signal potentiates motor system activity, which enhances the maximal level of voluntary force exertion (4).

The shout that was used to increase MVC in previous studies $(1,2)$ is also a form of "psyching up," a technique that can increase arousal to enhance physical performance with explosive force production (5). Psyching up refers to self-directed cognitive strategies used immediately prior to or during skill execution that are designed to enhance performance (6). Indeed, many athletes of tennis, power lifting, and other sports that require explosive movements undertake some form of psyching up in both training and competition $(2,6)$. However, the mechanisms underlying their enhancing effects on force production remain unknown. Listening to brief, loud sounds binaurally via headphones produces pupillary dilation (7), which suggests an increased level of arousal is achieved by enhancing sympathetic nerve system activity (8). Importantly, few previous studies have explored the influence of a shout on brain neuronal activity (i.e., the state of the pupil-linked neuromodulatory system and/or the motor system) with special reference to human maximal force exertion. 
Here, we investigated the influence of shouting on the pupil-linked neuromodulatory system state by examining pupil size $(9,10)$. We also examined the motor system state by examining motor evoked potentials (MEPs) in response to transcranial magnetic stimulation (TMS) applied over the contralateral primary motor cortex (M1), and by evaluating handgrip maximal voluntary force. Our results indicate that shouting can increase the handgrip force level of MVC through the reduction of motor cortical inhibition, possibly via the enhancement of pupil-linked neuromodulatory system activity. This study provides evidence that the muscular force-enhancing effect of shouting during maximal force exertion is related to both the motor system and the pupil-linked neuromodulatory system.

\section{Methods}

\section{Power Analysis}

We conducted an a priori power analysis to determine the required sample size for this experiment. We designed this experiment to have $80 \%$ power for detecting the effect sizes that we had previously found for the influence of motivational goal-priming on the motor system and action (0.46-0.64, Cohen's $d)(4$, 9 ) and/or pupil diameter $(0.50-0.61$, Cohen's $d)(9,10)$, using a significance level of $5 \%$. We used G*Power $3.1 \circledast$ (Institut für Experimentelle Psychologie, Düsseldorf, Germany) to compute the required sample size of the current study, which was 11 participants.

\section{Participants and Procedures}

Nineteen healthy Japanese right-handed individuals, as evaluated using the Edinburgh Handedness Inventory (11), participated in the study. There were 18 men and 1 woman, with a mean age \pm standard deviation of $20.4 \pm 2.0$ years. All participants provided both written and verbal informed consent according to the Declaration of Helsinki. The participants were university students with no clear description of strength training history, which suggests that they were untrained in exerting the maximal force generated briefly by a muscle or group of muscles at a specified speed. The experimental procedures complied with relevant laws and institutional guidelines and were approved by the Human Research Ethics Committee of the Faculty of Sport Sciences of Waseda University (approval number: 2017-253).

Experiments were designed to examine the influence of a self-generated shout on handgrip maximal voluntary force, pupillary size, and MEP in the flexor carpi ulnaris (FCU) muscle in response to TMS (see TMS for details). Each experiment consisted of two conditions (control and shout), each with a duration of approximately $216 \mathrm{~s}$, which included two phases: the experimental instruction phase, for about $26 \mathrm{~s}$, and the MVC task phase, for about $190 \mathrm{~s}$ (Fig. 1). The total experimental time was approximately 30 min. Participants received two conditions (control and shout), with a break of at least 15 min according to the experimental instructions on a screen in front of them (see Pupil Diameter Measurement for details). The order of the two conditions was counterbalanced so that 10 of the participants started with the control condition and the other participants started with the shout condition. In the shout condition, participants were asked to shout and squeeze a handgrip device with their maximal volition, whereas they were asked 
to never shout in the control condition. Both in the control and shout conditions, participants were asked to keep their head still and put their hands on their lap in a sitting posture as strictly as possible, maintaining stability in the core, irrespective of control or shout conditions. All experimental procedures were conducted automatically on a $60-\mathrm{Hz}$ cathode ray tube (CRT) screen that displayed the instructions in text form (see Pupil Diameter Measurement for details).

\section{Pupil Diameter Measurement}

Pupil diameters were measured using a TalkEye Lite system (Takei Scientific Instruments Co., Ltd., Tokyo, Japan). An image around the pupil was obtained by a camera employing near-infrared light-emitting diodes and a video graphics array $(640 \times 480)$ (digital signal processor built in) camera module (NCM03V, Nippon Chemi-Con Corporation, Tokyo, Japan). Banalization processing was performed on each image, and the pupil diameter was then measured according to the methods described by Wang et al. (12). Changes in pupil size were estimated by the area of the pupil $(9,10)$ while participants viewed the experimental instructions (the experimental instruction phase) and exerted handgrip MVC (the MVC task phase) in the control and shout conditions. We calculated the average pupil area from the onset of the first word presentation to the disappearance of the last word in the experimental instruction phase, and during each number of squeezing a handgrip device displayed for $5 \mathrm{~s}$ (see Handgrip Force Measurement) in the MVC task phase.

The following steps were taken to exclude the impact of experimenter expectations for participant responses and measurements as much as possible, and to objectively estimate the shout effect. 1) All experimental procedures were conducted automatically using a $60-\mathrm{Hz}$ CRT screen to display the text, and the experimental procedure was created using software designed for psychological experiments (Inquisit 3 Desktop Edition, Millisecond Software, Seattle, WA, USA). 2) All participants were instructed to follow the instructions on the screen only. 3) Pupil diameter measurements were automatically performed using the specially designed device with an eye-capturing camera to obtain the image around the pupil. Consequently, the paradigm used in the present study was less susceptible to experimenter bias than the outcome measurements that have typically been used when studying MVC (13).

All word stimuli were displayed in black $\left(20.8 \mathrm{~cd} / \mathrm{m}^{2}\right.$ : mean value of five measurements of luminance with an LS160 luminance meter; Konica Minolta, Inc., Tokyo, Japan) on a white screen $\left(124.2 \mathrm{~cd} / \mathrm{m}^{2}\right)$ during the experimental procedure. Immediately before the word presentation, the color of the screen was momentarily white without any black words. Thus, the pupil diameter transiently decreased because of the large increase in luminance offered by the white screen with maximum luminance $\left(129.6 \mathrm{~cd} / \mathrm{m}^{2}\right)$. We were unable to completely eliminate the possibility that this transient change in luminance affected pupil diameter; however, any effect should have been minimal because this phenomenon was common to all participants and conditions.

\section{Handgrip Force Measurement}


Force was measured using a handgrip device (KFG-5-120-C1-16, Kyowa Electronic Instruments, Tokyo, Japan). The experimental instruction on the screen asked participants to squeeze the handgrip device with the right (dominant) hand with his/her maximum effort when each number of " 1 st time" to " 5 th time" appeared, and to stop squeezing when the number disappeared. The handgrip device was fixed onto the right thigh by an elastic band so that the device did not move when it was squeezed by the participant. The number was displayed for $5 \mathrm{~s}$, and was repeated five times, with a 30-s inter-squeeze interval. The maximal values of the force exerted were averaged from the 500-ms steady state of force curve before each TMS to the 100ms state of force curve after each TMS according to the methods described by Gandevia et al. (14), across the five trials. This was defined as the handgrip MVC (Fig. 2A).

\section{TMS}

In both the control and shout conditions, single-pulse TMS was administered via a stimulator (M2002, Magstim, Whitland, UK) using a double figure-eight-shaped coil (4150-00 Double 70 mm Alpha Coil, Magstim) with a maximum magnetic field strength of $1.55 \mathrm{~T}$. Each participant sat upright with their elbows bent in front of them, resting on their thighs. The TMS coil was then positioned over the finger area of the left M1, which was determined as the area with the lowest resting motor threshold (rMT). This was defined as the area for which MEPs with peak-to-peak amplitudes greater than $50 \mu \mathrm{V}$ were induced in the FCU muscle $(9,15,16)$ in at least 5 out of 10 trials when participants were fully relaxed with their eyes closed (17). The coil position was stabilized throughout the experiment using a coil stand made from multiple products (Manfrotto Distribution KK, Tokyo, Japan). The optimal scalp position of M1 was marked directly onto the scalp with a black marker pen. The positioned coil was monitored continuously to maintain consistent positioning throughout the experiment. The rMTs ranged from $50-70 \%$ of the maximum stimulator output, and stimulus intensity for each participant was set at $110 \%$ of their rMT while viewing the experimental instruction. The stimulus intensity was set from $70-90 \%$ of the maximum stimulator output during handgrip force exertion. The stimulation was automatically delivered eight times at 3-s intervals during the experimental instruction phase. Thus, the MEP was recorded eight times for each condition (control or shout). Stimulations were manually delivered over the target site during each 5s MVC, with a 30-s inter-squeeze interval (Fig. 2A), the timing of TMS was different in each 5-s MVC in the MVC task phase. Thus, the MEP was recorded five times for each condition (control or shout). The TMS intensity was fixed for each participant. Surface electromyograms were obtained from the right FCU muscles via bipolar silver surface electrodes (bandpass, $15 \mathrm{~Hz}-10 \mathrm{kHz}$ ) using the tendon-belly method (4, 9).

\section{Background EMG and MEP Measurement and Analysis}

When the background EMG (bEMG) activity was high (Fig. 2B), it was difficult to discriminate the MEP in a single trace. We therefore calculated the averaged waveform of MEP (an average of eight recordings in the experimental instruction phase, and an average of five recordings in the MVC task phase for each condition evoked by TMS) to reduce the bEMG $(16,18)$. For each condition, we thus calculated the peakto-peak amplitude of the averaged MEP across eight recordings in the experimental instruction phase, 
and across five recordings in the MVC task phase. To measure the bEMG, a rectified EMG signal of the period $~ 100$ ms before TMS was integrated, during which the force was kept at the maximum force level (Fig. 2A, B). The duration of the cortical silent period was taken as the time interval from the stimulus artefact to the return of continuous EMG $(19,20)$ (Fig. 2C). When it was difficult to determine the end of the cortical silent period (because voluntary EMG activity does not recover abruptly, but rather recovers gradually), the end of the cortical silent period was determined when the corresponding rectified EMG activity reached a value within two standard deviations of the rectified EMG signal of the period $\sim 100 \mathrm{~ms}$ before TMS $(21,22)$.

\section{Statistical Analysis}

Statistically significant differences in the handgrip MVC, duration of the cortical silent period, and bEMG between the control and shout conditions were investigated using paired $t$-tests. MEP amplitude and pupil area were analyzed using repeated-measures two-way ANOVAs with within-participant factors of Condition (control or shout), and Phase (experimental instruction or MVC task). Greenhouse-Geisser corrections were applied when appropriate to adjust for non-sphericity, changing the degrees of freedom using a correction coefficient. Post hoc analysis used paired $t$-tests for each experimental condition (control or shout). A significance threshold of $P<0.05$ was chosen for all tests.

\section{Results}

\section{Handgrip Force}

The main finding was the enhancing effect of a shout on handgrip MVC (Fig. 3A). The handgrip MVC increased significantly from $259.7 \pm 16.9 \mathrm{~N}$ in the control condition to $304.4 \pm 16.6 \mathrm{~N}$ in the shout condition (Fig. 3B; $t(18)=-6.22, d=0.61 ; P=7.18 \times 10^{-6}$ ).

\section{MEP}

Compared with the control condition $(195.3 \pm 13.1 \mathrm{~ms})$, the duration of the cortical silent period during handgrip MVC was reduced in the shout condition $(179.3 \pm 11.4 \mathrm{~ms})$. The paired $t$-test revealed that this reducing effect of a shout was indeed significant (Fig. 4A, $\mathrm{t}(18)=2.68, d=0.30 ; P=0.0015$ ). However, there were no significant differences in MEP amplitudes between the two conditions during the experimental instruction (control: $170.0 \pm 33.7 \mu \mathrm{V}$; shout: $168.6 \pm 39.7 \mu \mathrm{V}$ ) or MVC task (control: $1125.8 \pm$ $75.1 \mu \mathrm{V}$; shout: $1149.3 \pm 79.8 \mu \mathrm{V}$ ) phases. A two-way analysis of variance (ANOVA) revealed a significant main effect of Phase $\left(F(1,18)=195.10 ; P=4.22 \times 10^{-11}\right.$; effect size: partial $\left.\eta 2=0.91\right)$, but no significant effect of Condition $(F(1,18)=0.18 ; P=0.67$; effect size: partial $\eta 2=0.01)$, and no interaction between Condition and Phase $(F(1,18)=0.17 ; P=0.68$; effect size: partial $\eta 2=0.01 ;$ Fig. 4B). Background electromyography $(E M G)$ revealed no significant changes among the conditions during the experimental instruction $(t(18)=-0.62, d=0.12 ; P=0.53)$ and MVC task $(t(18)=-1.03, d=0.10 ; P=0.31)$ phases. 
Figure 5A shows the time course of pupil area measurements from the onset of the experimental instruction phase to the end of the MVC task phase. Pupil size increased during the experimental instruction and MVC task phases for the two experimental (control or shout) conditions. Analysis revealed two significant main effects of Condition $\left(F(1,18)=30.12 ; P=3.26 \times 10^{-5}\right.$; effect size: partial $\eta^{2}$ $=0.62)$ and Phase $\left(F(1,18)=26.8 ; P=6.24 \times 10^{-5}\right.$; effect size: partial $\left.\eta^{2}=0.59\right)$, but no significant interaction between Condition and Phase $\left(F(1,18)=0.74 ; P=0.39\right.$; effect size: partial $\left.\eta^{2}=0.04\right)$. Post hoc analyses revealed a significantly larger change in pupil area in the shout condition than in the control condition during the experimental instruction $(t(18)=-3.11, d=0.42 ; P=0.006)$ and MVC task $(t(18)=-$ 5.03, $d=0.52 ; P=8.60 \times 10^{-5}$ ) phases (Fig. 5B).

\section{Discussion}

In the present study, we demonstrated that shouting significantly increased the handgrip force level of MVC, followed by an increase in pupil size and a reduction of the cortical silent period. Such an enhancing effect of shouting on handgrip MVC is generally consistent with results of previous studies (1, 2). Our findings indicate that the pupil-linked neuromodulatory system and the motor system are more excitable as a result of shouting, which leads to the production of additional muscular force in maximal exertion efforts. These results indicate that maximum volition-induced motor system activity does not drive muscles to produce the full force of which they are capable; that is, there is a latent ability for producing additional force that is hidden in ordinary force exertion.

\section{Pupil size as a fine index of actual intensity of the handgrip contraction}

Pupillometry has long been used as a measure of brain state. A number of studies

have reported that pupillary dilation is related to mental effort (cognitive load), and the correspondence between cognitive load and pupillary dilation has been documented in several contexts, including pairedassociate learning (23). The pupil size is well known to increase according to the complexity of the mental task (23). A recent study has demonstrated that the pupil size also increases during physical effort, the degree of which reflects the actual intensity of muscular contraction (24). Thus, we examined pupil size during handgrip MVC between the shout and control conditions. Because we consider that the effects of noradrenaline (NE) on the activity of motor cortical neurons as described below is indirectly estimated by pupil size, which is also supported by some previous studies (25-27).

\section{Enhancing effect of shouting on motor system activity through the potentiation of the pupil-linked neuromodulatory system}


Shouting significantly increased pupil size and reduced the cortical silent period. Changes in pupil diameter are considered to correspond to the activity of neuromodulators, including NE and acetylcholine, which produce alterations in the brain state and corresponding changes in behavior. It is currently unknown whether activity in only noradrenergic locus coeruleus (LC) neurons directly influences pupil size; however, noradrenergic neurons are active during pupillary dilation (28).

The effects of NE on the activity of motor cortical neurons are presumed to be as follows. First, NE increases the excitability of large pyramidal cells in layer $\mathrm{V}$ of the rat motor cortex (29). Second, NE depresses evoked inhibitory postsynaptic potentials in the rat sensorimotor cortex through the presynaptic inhibition of gamma-aminobutyric acid (GABA)ergic interneurons $(30,31)$. Third, in pyramidal cells in layer $\mathrm{V}$ of the rat prefrontal cortex, NE increases the frequency of spontaneous excitatory postsynaptic potentials (32-34). These enhancing effects of NE on the activity of motor cortical neurons result in increased intracortical facilitation and reduced intracortical inhibition. Such enhancing effects of $\mathrm{NE}$ on the activity of motor cortical neurons results in a reduced duration of the cortical silent period, as was observed in the current study (Fig. 4A). Although our experimental design did not allow the direct assessment of intracortical inhibition, changes in silent periods of longer than $100 \mathrm{~ms}$, as recorded in the hand muscles of healthy participants (35), are considered an index of cortical inhibition (19). The site of origin of the cortical silent period is largely in the M1 (19), in which GABABergic circuits may generate the cortical silent period (36-38). Thus, shouting is likely to reduce inhibition in the M1, which enhances motor excitability.

Despite the aforementioned enhancing effect of NE on motor cortical activity, we failed to detect any changes in MEP amplitude during MVC; there were no significant differences in MEP amplitudes between the shout and control conditions (Fig. 4B). This failure to detect any change might be associated with recruitment of the M1 neurons to reach a plateau level during MVC. In other words, most of the M1 neurons had already been recruited (39); thus, fewer neurons were available to respond to TMS. Thus, the M1 neurons recruitment to reach a plateau level during MVC might have overshadowed any differences in MEP amplitudes between the shouting and control conditions.

\section{Relationship between shouting and pupillary dilation}

The production of 'shouting' necessitates two pathways, which are organized hierarchically, building from the basic levels in the lower brain stem and spinal cord to the most

complex levels in the anterior cingulate cortex (ACC) and the laryngeal motor cortex (LMC), respectively (40). The limbic vocal control pathways responsible for the control of innate nonverbal and emotional vocalizations, and the laryngeal motor cortical pathway modulates the fine motor control of voluntary voice production-such as speech and song-as well as the voluntary production of innate vocalizations. Coordination and interactions between the LMC and ACC-periaqueductal gray (PAG) pathways are indispensable for proper voice control and voice initiation in the shout condition. In the present study, participants in the shout condition were asked to shout and perform handgrip MVC (see Participants and 
Procedures). The LMC requires inputs from the inferior frontal gyrus for motor planning of voice production, and from the supplementary motor area to prepare for vocal motor command execution. In the limbic vocal control pathway, the PAG mainly plays a gating role in triggering a vocal response and regulating its intensity, whereas the ACC is involved in the voluntary control of voice initiation and its emotional intonation. Thus, coordination and interactions between the LMC and ACC-PAG pathways are invaluable for the self-generating shout.

The LMC does not only connect reciprocally with motor cortices, such as the surrounding ventral and dorsal premotor cortices, primary motor cortices, and supplementary motor area, but also with subcortical regions, such as the claustrum, ventral and mediodorsal thalamus, medial parabrachial nucleus, deep mesencephalic nucleus, and LC (40). The PAG receives direct projections from the ACC as well as from other cortical and subcortical regions that control the limbic, sensory, motor, cognitive, and arousal systems (41). The PAG receives noradrenergic projections from the brain stem reticular formations (e.g., the LC, ventrolateral medulla [A1], and dorsal pons [5th]). Thus, shouting may stimulate the activity of LC neurons, possibly by enhancing the activity of the LMC and ACC-PAG pathways, which results in pupillary dilation.

Pupillary dilation was not observed during the MVC task phase, but also during the experimental instruction phase. The cause of pupillary dilation during the experimental instruction phase is assumed to be as follows. First, pupillary dilation during this phase may be caused by motor imagery, performing the handgrip MVC combined with self-generated shout immediately after the instruction was given. Indeed, when debriefed, participants reported that they imagined the combined motor action. Some previous studies have reported such a motor imagery-induced pupillary dilation (42-44). Although each motor action (i.e., handgrip or shout) is essentially primitive, the combined motor action execution requires the processing of higher-order motor control (see the previous descriptions of central shouting control). Thus, motor imagery during the instruction phase may induce pupillary dilation. Another cause may be related to the time pressure associated with the motor imagery: the participants had to complete the rehearsal activity before the text stating " 1 st time" was unpredictably displayed on the monitor, instructing the participants to start squeezing the handgrip device immediately after the experimental instruction. Some previous studies reported that such time pressure is inherent in the structure of a mental task and induces particularly large pupillary dilations $(42,45)$. Thus, time pressure associated with motor imagery may have induced pupillary dilation in the current study.

Consequently, we cannot exclude the possibility that pupil-dilating effects during the experimental instruction phase might influence pupillary dilation during the handgrip MVC phase with shouting. However, the percentage increase in pupillary dilation during MVC $(12.1 \% \pm 2.7 \%)$ was much greater than that during experimental instruction $(8.5 \% \pm 2.9 \%)$. Thus, a change in pupillary dilation during the experimental instruction phase cannot adequately explain the difference in pupillary dilation across tasks. We therefore consider that the pupillary dilations in the experimental instruction and MVC task phases had different main causes. 


\section{Conclusion}

We note that shouting led to dilated pupils and reduced the cortical silent period during MVC, which increased handgrip maximal voluntary force levels. The increased MVC may be caused by the reduction of motor cortical inhibition via potentiation of the pupil-linked neuromodulatory system. These results indicate that maximum volition-induced motor system activity does not drive muscles to produce the full force of which they are capable. In turn, this suggests that a fluctuating factor of MVC is an active characteristic of human brain neural systems; maximum volition does not cause the motor system to produce maximum activity. However, it remains unknown why the human brain neural system might have such an 'inhibiting mechanism' during muscular force exertion with maximal volition.

\section{Declarations}

\section{Acknowledgments}

This work was supported by a Grant-in-Aid for Scientific Research (C) from the Ministry of Education, Culture, Sports, Science and Technology in Japan (Projects 21K11532).

\section{Author Contributions:}

The complete list of author contributions to the paper according to the CRediT model is as follows: 1.Conceptualisation Y.T.; 2.Data curation Y.T.; 3.Formal analysis Y.T. and D.N. (supporting); 4.Grant acquisition Y.T.; 5.Investigation Y.T.; 6.Methodology Y.T.; 7.Project administration Y.T.; 8.Resources Y.T.; 9.Software Y.T. and D.N.; 10.Supervision Y.T.; 11.Validation Y.T.; 12.Visualisation Y.T.; 13.Writing original draft Y.T.; 14. Writing-review and editing Y.T. and D.N (supporting).

\section{Competing Interest Statement:}

The authors declare no competing interests.

\section{References}

1. Ikai, M. \& Steinhaus, A. H. Some factors modifying the expression of human strength. Appl. Physiol. $16,157-163$ (1961).

2. Welch, A. S. \& Tschampl, M. Something to shout about: a simple, quick performance enhancement technique improved strength in both experts and novices. Appl. Sport Psychol. 24, 418-428 (2012).

3. McNair, P. J., Depledge, J., Brettkelly, M. \& Stanley, S. N. Verbal encouragement: effects on maximum effort voluntary muscle action. J. Sports Med. 30, 243--245 (1996).

4. Takarada, Y. \& Nozaki, D. Maximal voluntary force strengthened by the enhancement of motor system state through barely visible priming words with reward. PLOS ONE 9, e109422 (2014). 
5. Perkins, D., Wilson, G. V. \& Kerr, J. H. The effects of elevated arousal and mood on maximal strength performance in athletes. Appl. Sport Psychol. 13, 239-259 (2001).

6. Tod, D., Iredale, F. \& Gill, N. 'Psyching-up' and muscular force production. Sports Med. 33, 47-58 (2003).

7. DiGirolamo, G. J., Patel, N. \& Blaukopf, C. L. Arousal facilitates involuntary eye movements. Brain Res. 234, 1967-1976 (2016).

8. Bradley, M. M., Miccoli, L., Escrig, M. A. \& Lang, P. J. The pupil as a measure of emotional arousal and autonomic activation. Psychophysiology 45, 602-607 (2008).

9. Takarada, Y. \& Nozaki, D. Motivational goal-priming with or without awareness produces faster and stronger force exertion. Rep. 8, 10135 (2018).

10. Takarada, Y. \& Nozaki, D. Pupil dilations induced by barely conscious reward goal-priming. Neuropsychologia 103, 69-76 (2017).

11. Oldfield, R. C. The assessment and analysis of handedness: the Edinburgh inventory. Neuropsychologia 9, 97-113 (1971).

12. Wang, J., Zhang, G. \& Shi, J. Pupil and glint detection using wearable camera sensor and nearinfrared LED array. Sensors 15, 30126-30141 (2015).

13. Nuzzo, J. L., Taylor, J. L. \& Gandevia, S. C. CORP: Measurement of upper and lower limb muscle strength and voluntary activation. Appl. Physiol. 126, 513-543 (2019).

14. Gandevia, S. C., Allen, G. M., Butler, J. E. \& Taylor, J. L. Supraspinal factors in human muscle fatigue: evidence for suboptimal output from the motor cortex. Physiol. 490, 529-536 (1996).

15. Takarada, Y., Mima, T., Abe, M., Nakatsuka, M. \& Taira, M. Inhibition of the primary motor cortex can alter one's "sense of effort": effects of low-frequency rTMS. Res. 89, 54-60 (2014).

16. Takarada, Y., Ohki, Y. \& Taira, M. Effect of transient vascular occlusion of the upper arm on motor evoked potentials during force exertion. Res. 76, 224-229 (2014).

17. Rossini, P. M., et al., Non-invasive electrical and magnetic stimulation of the brain, spinal cord and roots: basic principles and procedures for routine clinical application. Report of an IFCN committee. Clin. Neurophysiol. 91, 79-92 (1994).

18. Shibuya, S. \& Ohki, Y. Cutaneous inputs can activate the ipsilateral primary motor cortex during bimanual sensory-driven movements in humans. Neurophysiol. 92, 3200-3209 (2004).

19. Inghilleri, M., Berardelli, A., Cruccu, G. \& Manfredi, M. Silent period evoked by transcranial stimulation of the human cortex and cervicomedullary junction. Physiol. 466, 521-534 (1993).

20. Damron, L. A., Dearth, D. J., Hoffman, R. L. \& Clark, B. C. Quantification of the corticospinal silent period evoked via transcranial magnetic stimulation. Neurosci. Methods 173, 121-128 (2008).

21. Duclay, J., Pasquet, B., Martin, A \& Duchateau, J. Specific modulation of spinal and cortical excitabilities during lengthening and shortening submaximal and maximal contractions in plantar flexor muscles. Appl. Physiol. 117, 1440-1450 (2014). 
22. Groppa, S., et al., A practical guide to diagnostic transcranial magnetic stimulation: report of an IFCN committee. Neurophysiol. 123, 858-882 (2012).

23. Kahneman, D. Toward a theory of mental effort: Attention and Effort Ch. 3, (Prentice-Hall, New Jersey, 1973).

24. Zénon, A., Sidibé, M. \& Olivier, E. Pupil size variations correlate with physical effort perception. Behav. Neurosci. 8, 286 (2014).

25. Joshi, S., Li, Y., Kalwani, R. \& Gold, J. I. Relationships between pupil diameter and neuronal activity in the locus coeruleus, colliculi, and cingulate cortex. Neuron 89, 221-234 (2016).

26. Reimer, J., et al., Pupil fluctuations track rapid changes in adrenergic and cholinergic activity in cortex. Commun. 7, 13289 (2016).

27. Murphy, P. R., O'Connell, R. G., O’Sullivan, M., Robertson, I. H. \& Balsters, J. H. Pupil diameter covaries with BOLD activity in human locus coeruleus. Brain Mapp. 35, 4140-4154 (2014).

28. Larsen, R. S. \& J. Waters, J. Neuromodulatory correlates of pupil dilation. Front. Neural Circuits 12, 21 (2018).

29. Foehring, R. C., Schwindt, P. C. \& Crill, W. E. Norepinephrine selectively reduces slow Ca2+- and $\mathrm{Na}+-$ mediated K+ currents in cat neocortical neurons. Neurophysiol. 61, 245-256 (1989).

30. Bennett, B. D., Huguenard, J. R. \& Prince, D. A. Adrenergic modulation of GABAA receptor-mediated inhibition in rat sensorimotor cortex. Neurophysiol. 79, 937-946 (1998).

31. Bennett, B. D., Huguenard, J. R. \& Prince, D. A. Adrenoceptor-mediated elevation of ambient GABA levels activates presynaptic GABA(B) receptors in rat sensorimotor cortex. Neurophysiol. 78, 561566 (1997).

32. Marek, G. J. \& Aghajanian, G. K. 5-HT2A receptor or alpha1-adrenoceptor activation induces excitatory postsynaptic currents in layer $\mathrm{V}$ pyramidal cells of the medial prefrontal cortex. $J$. Pharmacol. 367, 197-206 (1999).

33. Ziemann, U., Lönnecker, S., Steinhoff, B. J. \& Paulus, W. The effect of lorazepam on the motor cortical excitability in man. Brain Res. 109, 127-135 (1996).

34. Ziemann, U., Chen, R., Cohen, L. G. \& Hallett, M. Dextromethorphan decreases the excitability of the human motor cortex. Neurology 51, 1320-1324 (1998).

35. Cantello, R., Gianelli, M., Civardi, C. \& Mutani, R. Magnetic brain stimulation: the silent period after the motor evoked potential. Neurology 42, 1951-1959 (1992).

36. Connors, B. W., Malenka, R. C. \& Silva, L. R. Two inhibitory postsynaptic potentials, and GABAA and GABAB receptor-mediated responses in neocortex of rat and cat. Physiol. 406, 443-468 (1988).

37. Werhahn, K. J., Kunesch, E., Noachtar, S., Benecke, R. \& Classen, J. Differential effects on motorcortical inhibition induced by blockade of GABA uptake in humans. Physiol. 517(Pt 2), 591597 (1999).

38. Siebner, H. R., Dressnandt, J., Auer, C. \& Conrad, B. Continuous intrathecal baclofen infusions induced a marked increase of the transcranially evoked silent period in a patient with generalized dystonia. 
Muscle Nerve. 21, 1209-1212 (1998).

39. Matthews, P. B. C. The effect of firings on the excitability of a model motoneurones and its implications for cortical stimulation. Physiol. 518, 867-882 (1999).

40. Simonyan, K. \& Horwitz, B. Laryngeal motor cortex and control of speech in humans. Neuroscientist 17, 197-208 (2011).

41. Dujardin, E. \& Jurgens, U. Afferents of vocalization-controlling periaqueductal regions in the squirrel monkey. Brain Res. 1034, 114-131 (2005).

42. Simpson, H. M. \& Paivio, A. Changes in pupil size during an imagery task without motor response involvement. Sci., 5, 405-406 (1966).

43. White, O. \& French, R. M. Pupil diameter may reflect motor control and learning. Mot. Behav., 49, 141149 (2017).

44. Carey, K., Moran, A. \& Rooney, B. Learning choreography: An investigation of motor imagery, attentional effort, and expertise in modern dance. Psychol. 10, 1-11 (2019).

45. Simpson, H. M. \& Paivio, A. Effects on pupil size of manual and verbal indicators of cognitive task fulfillment. Psychophys. 3, 185-190 (1968).

\section{Figures}

Iinstruction

(26.7s)

Grip strength

MEP

Pupil area

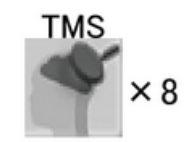

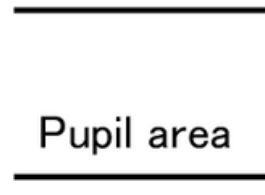

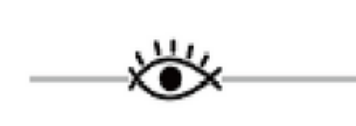

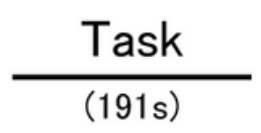

MVC

$(30 \mathrm{~s})$

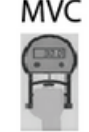

$(30 \mathrm{~s})$

MVC

TMS

TMS

TMS
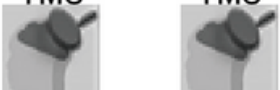

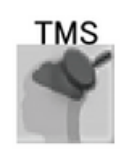

(30 s)
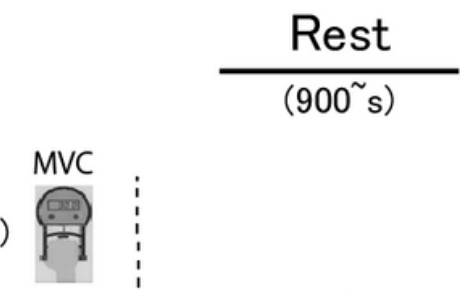

$(900 \sim s)$
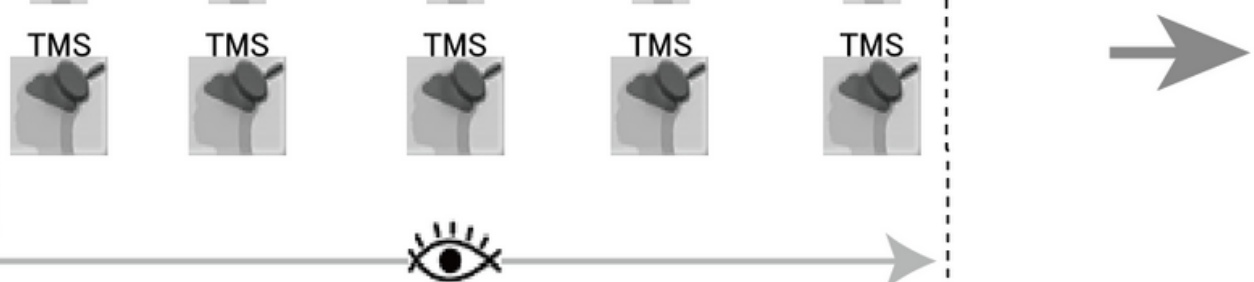

Figure 1

Experimental procedure. Each experiment consists of two conditions (control and shout in MVC task phase), each condition consists of two (experimental instruction and MVC task) phases. Each participant performed two (control and shout) conditions, with a break of at least 15 min between each. Total experimental time was approximately $30 \mathrm{~min}$. Instruction: experimental instruction phase. Task: MVC task phase. 
A

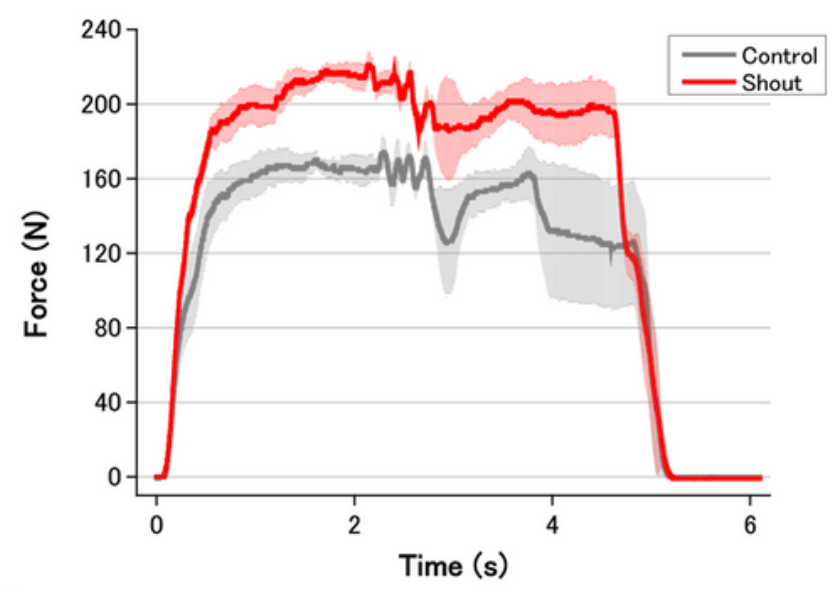

B

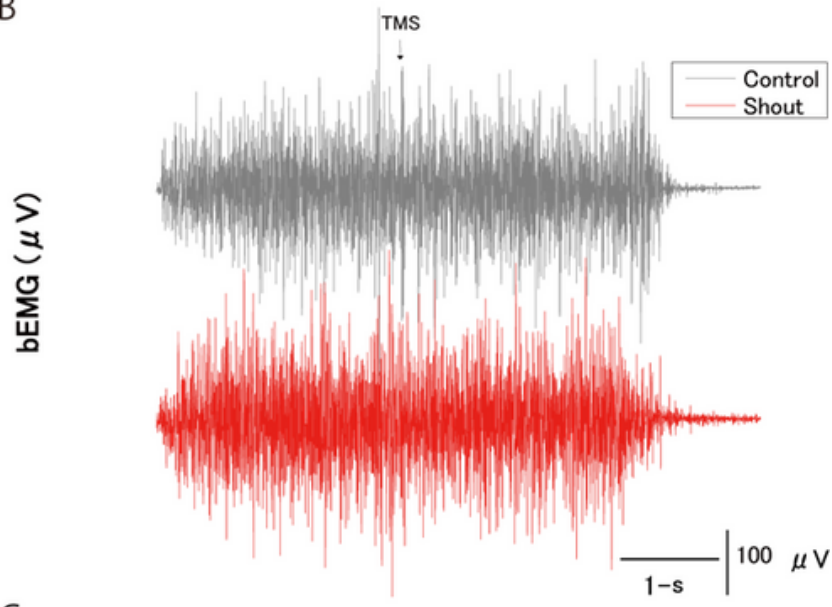

C

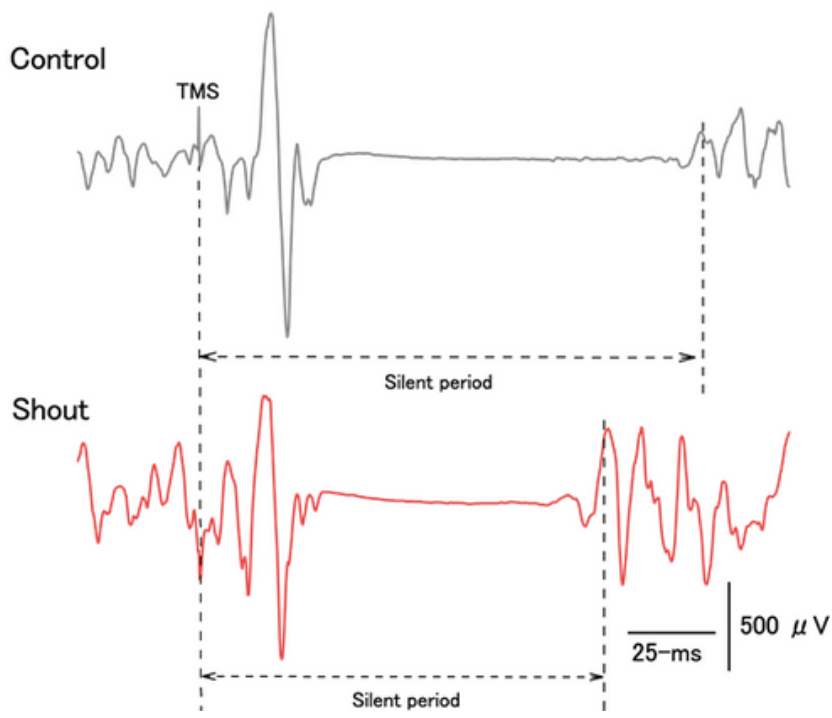

Figure 2

Typical recordings of handgrip force, background electromyography (bEMG), and typical motor evoked potential (MEP) waveforms of the flexor carpi ulnaris during the maximal voluntary contraction force (MVC) of handgrip in each experimental condition (control or shout) in a single participant. The timing of transcranial magnetic stimulation is indicated by the arrow. The handgrip force declined when TMS was delivered during the contraction, the timing of which was different in each contraction. (A) Data of force 
are expressed as the mean \pm standard error of the mean of five recordings. (B) bEMG in a handgrip contraction for each condition. (C) Duration of the cortical silent period in a handgrip contraction for each condition.

A

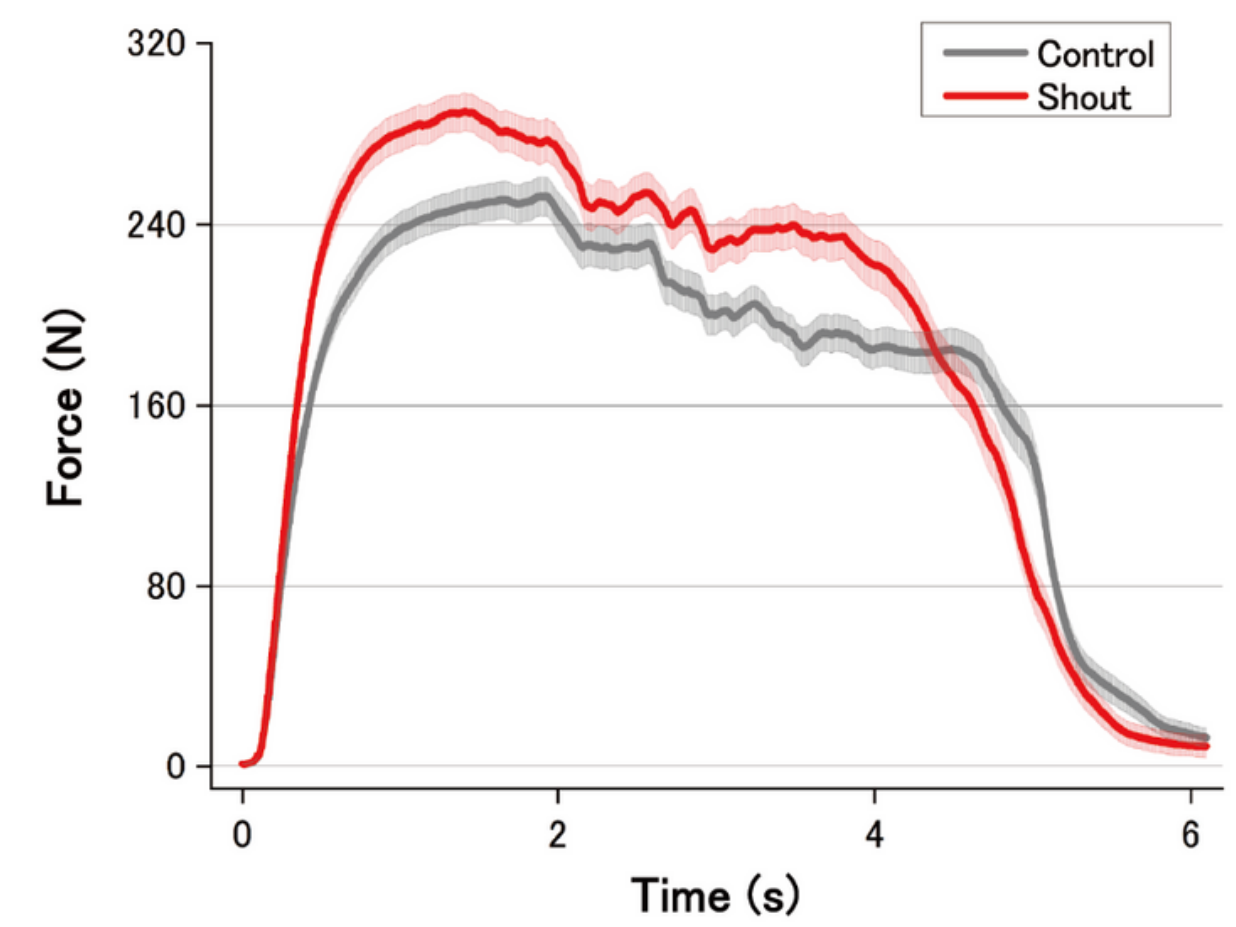

B

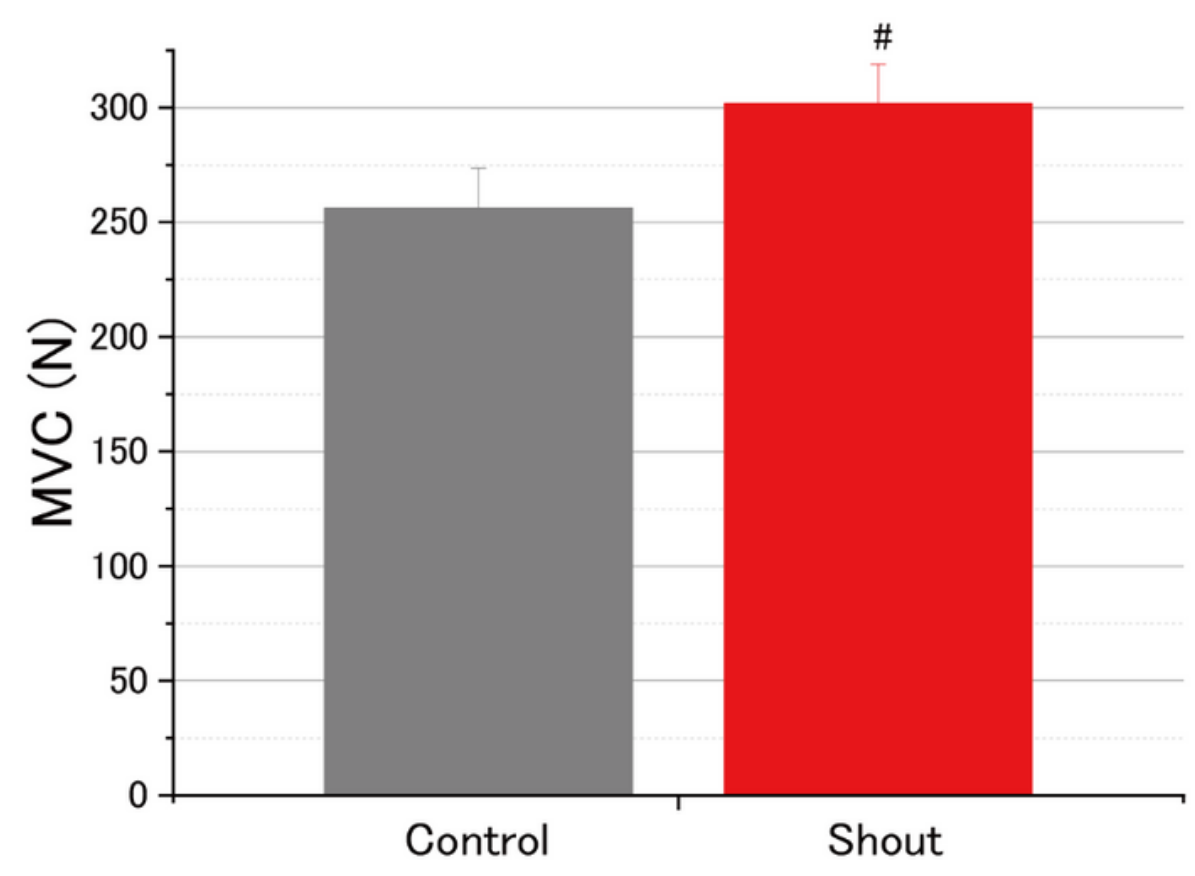

Figure 3

Effects of shouting on the maximal voluntary contraction force (MVC) of handgrip. (A) Typical recordings of handgrip force in each experimental condition (control or shout), which were averaged across all 
participants. (B) The averaged MVC across the five handgrip trials for the two conditions. The shout condition resulted in a greater handgrip MVC compared with the control condition. Data are expressed as the mean \pm standard error of the mean (\#P $<0.01$, paired t-test).

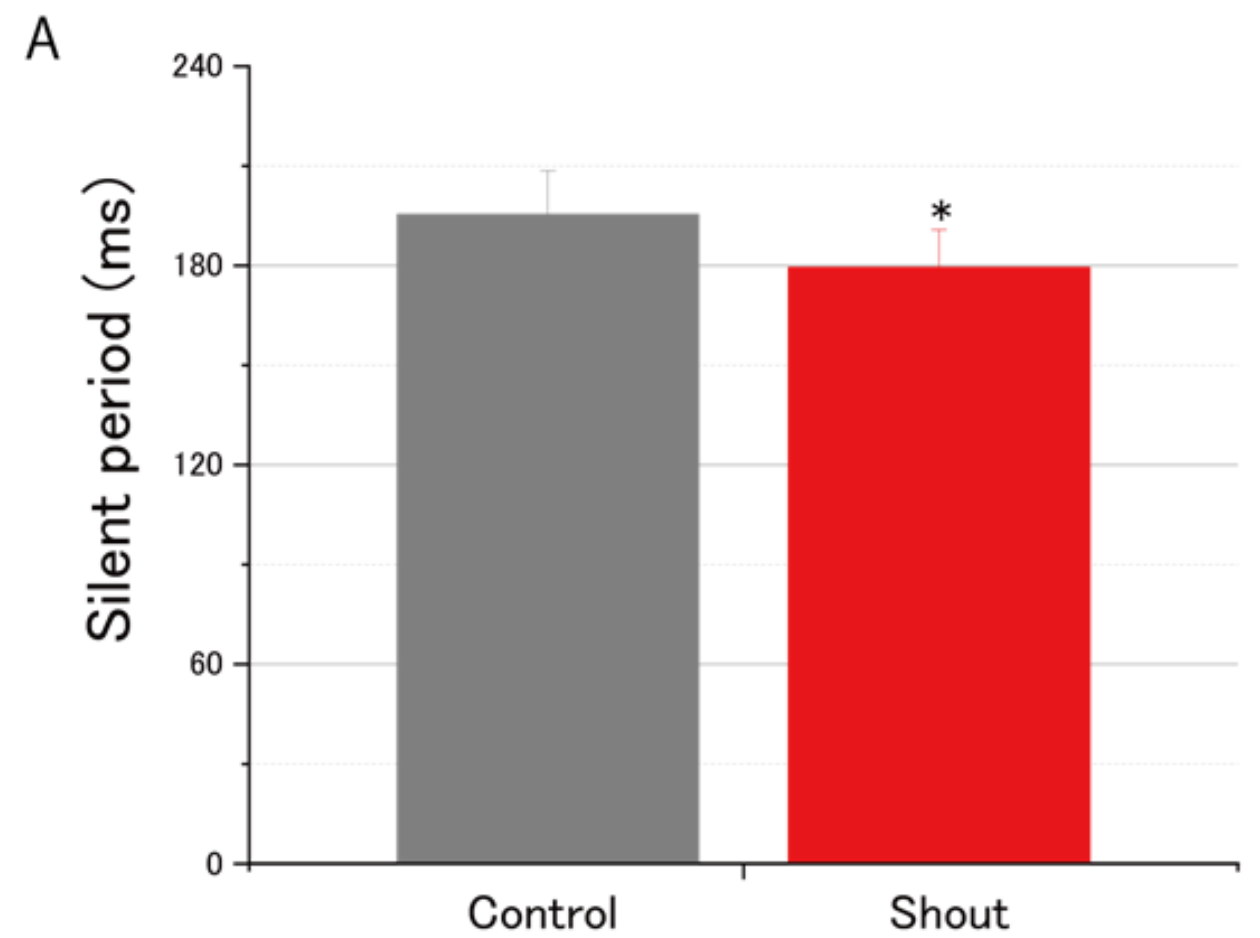

B

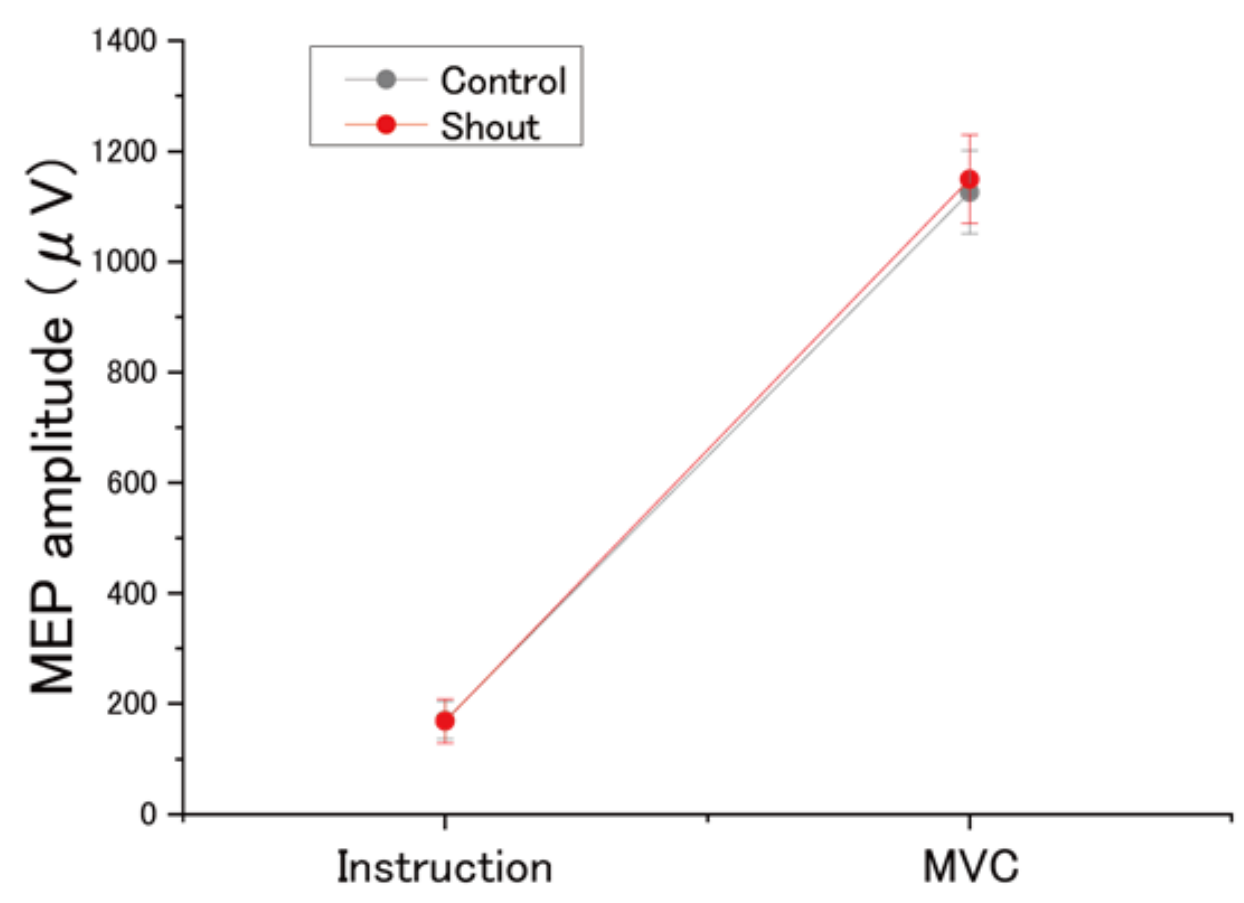

Figure 4

Effects of shouting on the cortical silent period and motor evoked potential (MEP) amplitude. (A) Durations of the cortical silent period for the two conditions during the maximal voluntary contraction 
force (MVC) of handgrip. The duration of the cortical silent period in the shout condition was significantly shorter than in the control condition ( ${ }^{*} \mathrm{P}<0.05$, paired t-test). (B) Amplitudes of MEPs of the flexor carpi ulnaris during the experimental instruction and MVC task phases for the two experimental conditions (control or shout). There was no significant difference in MEP amplitude between the two conditions. Data are expressed as the mean \pm standard error of the mean.

A

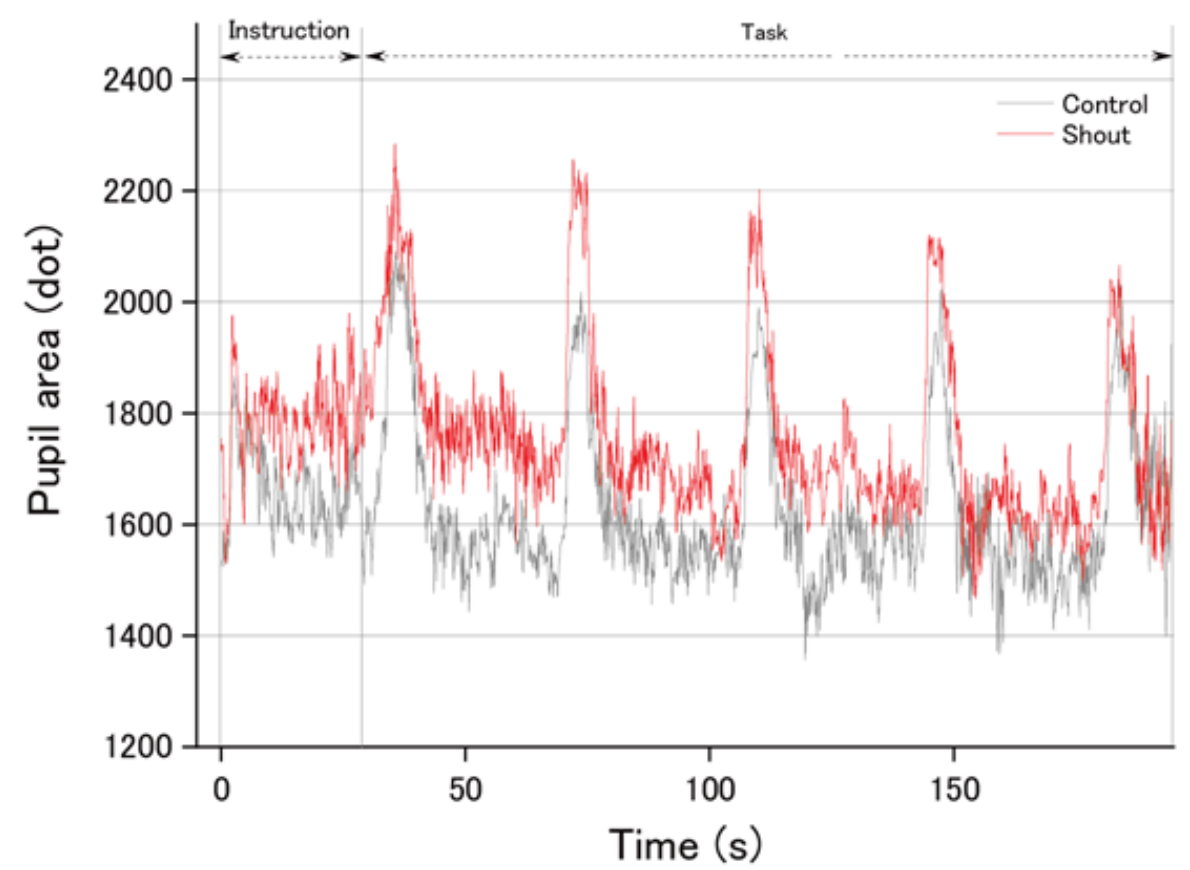

B

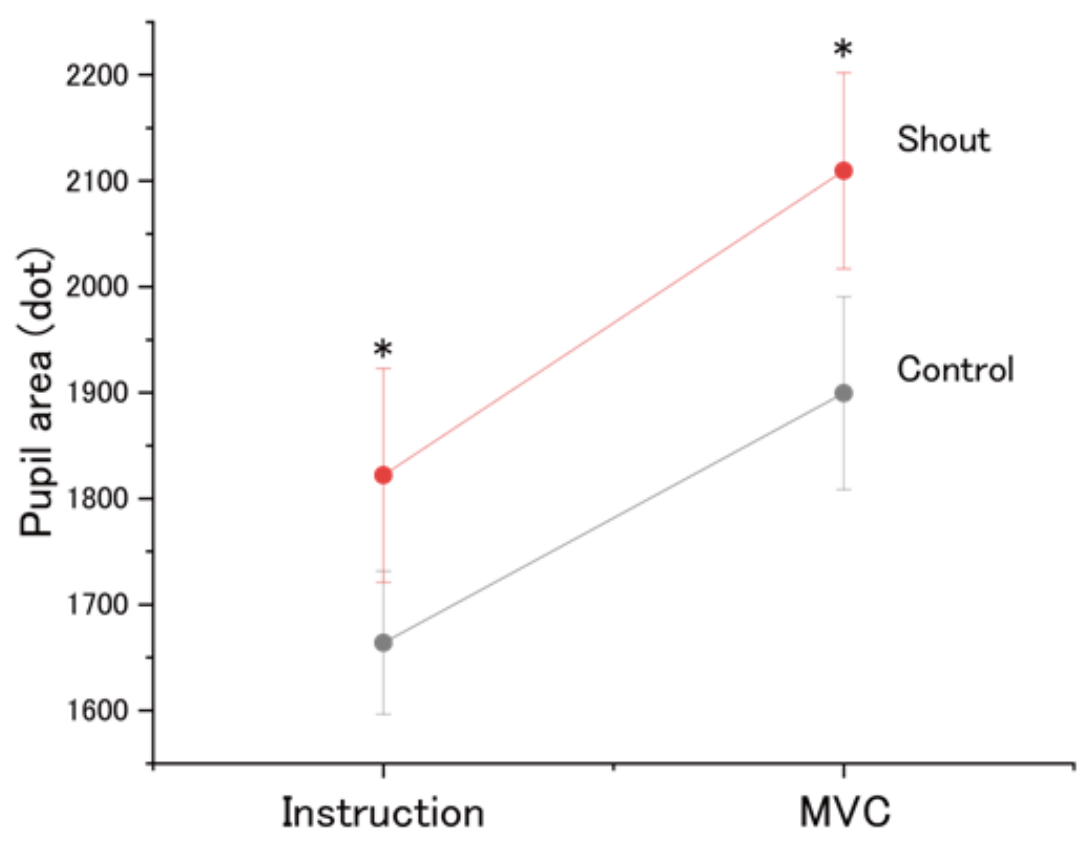

Figure 5 
Effects of shouting on pupil area over time. (A) Pupil area (dots) starting at the onset of word presentation in the experimental procedure and lasting until the end of the maximal voluntary contraction force (MVC) task. Pupil area is expressed as the mean for each experimental condition (control or shout). The data were low-pass filtered with a cut-off frequency of $1 \mathrm{~Hz}$ using a fourth-order Butterworth filter. Two bidirectional arrows $(\leftrightarrow)$ indicate the period of the experimental instruction and the period of the MVC handgrip task, respectively. Instruction: experimental instruction phase. Task: MVC task phase. Pupil area (dots) is expressed as the mean during the period of the experimental instruction phase and during MVC (not during the MVC task phase) for each experimental condition (control or shout). (B) Pupil area (dots) during the period of the experimental instruction phase and during each number of squeezing a handgrip device displayed for $5 \mathrm{~s}$ (not during the MVC task phase) for the two experimental conditions (control or shout). The shout condition resulted in a greater pupil area compared with the control condition. Pupil area data are expressed as the mean \pm standard error of the mean. ${ }^{*} P<0.05$, vs. control condition. 